\title{
Clinical features of central nervous system infections and experience in differential diagnosis from neuropsychiatric lupus erythematosus in a cohort of 8491 patients with systemic lupus erythematosus
}

\author{
Mengdi Jiang ${ }^{1}$, Xiaochun Shi ${ }^{2}$, Xin $\mathrm{GaO}^{3}$, Jingwen Niư ${ }^{4}$ Xiaomin $\mathrm{Hu}^{5}$, Lidan Zhao ${ }^{1 *}$ and Xuan Zhang ${ }^{1 *}$ (D)
}

\begin{abstract}
Background: In clinical practice, discrimination between central nervous system (CNS) infections in patients with systemic lupus erythematosus (SLE) and neuropsychiatric lupus erythematosus (NPSLE) could be urgent and critical yet extremely challenging. Given this, this study aimed to investigate the clinical features and outcomes of infections in the CNS in patients with SLE and to establish a simplified scoring system for guiding the discrimination of CNS infections from NPSLE.

Methods: A total of 95 patients who were identified as having CNS infections among 8491 SLE patients between January 1992 and January 2018 were included in this retrospective study. NPSLE patients admitted at the same period were randomly selected for comparison. Key factors either clinically valuable or statistically significant for discriminating CNS infections from NPSLE were integrated to build a simplified scoring system. Another group of 22 SLE patients complicated with suspected newly onset of CNS infections or NPSLE admitted after January 2018 was enrolled to verify the utility of the scoring system.

Results: Sixty-three positive pathogens were identified in 59 patients of the total 95 CNS infection cases. Compared with the NPSLE group, the CNS infections group had a longer disease duration (21.0 [3.0-50.0] vs. 1.0 [0-22.0] months, $P<0.05)$, exhibited more fever $(96.8 \%$ vs. $23.2 \%, P<0.001)$ and polymorphonuclear leukocyte leukocytosis in the cerebrospinal fluid (CSF) $(45.6 \%$ vs. $0.5 \%, P<0.05)$, and had significantly decreased CSF glucose $(2.0 \pm 1.3$ vs. $3.3 \pm 0.9 \mathrm{mmol} / \mathrm{L}, P<0.01$ ), whereas hypocomplementemia seemed to be a strong hint of NPSLE (44.6\% vs. $77.4 \%$, $P<0.001)$. A simplified scoring system integrated with 8 key factors was established for guiding clinical differential diagnosis. By setting the cutoff value at 4 and verifying in a group of SLE patients complicated with newly occurred suspected CNS infection or NPSLE, a sensitivity of $85.7 \%$ and specificity of $93.3 \%$ with the area under the curve (AUC) being 0.93 (95\% Cl 0.80-1.00) were obtained.
\end{abstract}

Conclusions: CNS infections are a fatal complication of SLE and can be difficult to discriminate from NPSLE. A simplified scoring system may help to make preliminary discrimination of CNS infections from NPSLE.

Keywords: Central nervous system, Infection, Systemic lupus erythematosus, Risk factor, Scoring system

\footnotetext{
*Correspondence: zhaolidan@hotmail.com; zxpumch2003@sina.com 'Department of Rheumatology and Clinical Immunology, Peking Union Medical College Hospital, Clinical Immunology Center, Chinese Academy of Medical Sciences and Peking Union Medical College, The Ministry of Education Key Laboratory, Beijing, China

Full list of author information is available at the end of the article
}

(c) The Author(s). 2019 Open Access This article is distributed under the terms of the Creative Commons Attribution 4.0 International License (http://creativecommons.org/licenses/by/4.0/), which permits unrestricted use, distribution, and reproduction in any medium, provided you give appropriate credit to the original author(s) and the source, provide a link to the Creative Commons license, and indicate if changes were made. The Creative Commons Public Domain Dedication waiver (http://creativecommons.org/publicdomain/zero/1.0/) applies to the data made available in this article, unless otherwise stated. 


\section{Background}

Systemic lupus erythematosus (SLE) is a typical autoimmune disorder characterized by widespread immune deregulation, resulting in systemic inflammation and multi-organ impairments. Immunosuppressive agents, including glucocorticoids (GCs) and disease-modifying antirheumatic drugs (DMARDs), are the main therapeutic tools but may increase the risk of severe infections [1-4]. Infections are one of the major causes of morbidity and mortality in SLE patients. According to previous studies, infections are estimated to be responsible for 11 to $50 \%$ of deaths in SLE [5-8]. In clinical practice, discrimination between infections and SLE flares could be extremely challenging, for example, infections may mimic symptoms of SLE, leading to confusion over the diagnosis and delay of treatment. Central nervous system (CNS) infections constitute up to 3\% of all infections in lupus patients, and although not very common, these infections are life-threatening and severely disabling [9]. More importantly, CNS infections in SLE patients are easily confused with neuropsychiatric lupus erythematosus (NPSLE) which is a challenging complication of SLE that requires vigorous treatment with highdose GCs and DMARDs. These two conditions may exhibit similar symptoms and phenotypes, yet they require completely different therapeutic strategies. CNS infections usually have a high mortality rate unless an accurate diagnosis is promptly made and the appropriate therapeutic intervention is initiated at the very early stage.

To better understand and manage CNS infections in patients with SLE, we conducted this study in 8491 SLE in-patients admitted in the past 26 years to Peking Union Medical College Hospital (PUMCH), which is the tertiary referral center in China. In this study, we aim to not only identify the clinical features and etiology of CNS infections, but also provide the diagnostic clues for discriminating CNS infections from NPSLE among SLE patients. To our knowledge, this is the largest cohort study in this field to date and may provide practical guidance for clinicians to distinguish these two severe and confusing complications.

\section{Methods}

\section{Patient selection}

PUMCH in-patient register database system was used for two-step screening electronically. First, we screened 8491 in-patients with a final diagnosis of "systemic lupus erythematosus" who were consecutively admitted to PUMCH from January 1992 to January 2018. Then, to identify probable cases of CNS infections, we searched the patient records for keywords including the following: "central nervous system infection," "intracranial infection," "encephalitis," "meningitis," "meningoencephalitis," and "brain abscess." To identify probable cases of NPSLE, we searched the patient records for keywords including the following: "lupus encephalopathy" and "neuropsychiatric lupus." Patients with NPSLE would constitute the pool of candidates for the control group. Ninety-five SLE patients were confirmed to have CNS infections according to the criteria mentioned below. An equal number of NPSLE patients were randomly selected, using a computational algorithm that matched the age and gender. Medical records of these cases were reviewed, and those lacked key information were removed from the study. Relevant information was collected, and uncertain cases were discussed by a multiple disciplinary team (MDT) consisting of two rheumatologists, one specialist of infectious diseases, one radiologist, and one neurologist. If the MDT could not come to a consensus on a particular case, that case would be excluded from the study. If a patient in the NPSLE group had a concurrent infection, they were also excluded from the study. Further details of the screening process are shown in Additional file 1. All patients fulfilled the 1982 American College of Rheumatology (ACR) classification criteria for SLE. NPSLE refers to the neurologic and psychiatric syndromes involving CNS categorized by ACR subcommittee in 1999 [10] with excluding causes other than lupus. The definitive diagnosis of CNS infections was based on (1) clear etiological evidence, including positive finding of the pathogens from cerebrospinal fluid (CSF) or brain lesion biopsy via microorganism culture or smear with gram, acid-fast, or India ink staining; (2) indirect etiological evidence, including positivity in pathogen antigen/ antibody detection, such as the cryptococcal antigen latex agglutination system (CALAS) test, and Cysticercus cellulosae antibody detection, or positivity for pathogen DNA detected by polymerase chain reaction (PCR); (3) clinical diagnosis confirmed by expert opinions based on comprehensive evaluation of clinical manifestations, CNS examinations, laboratory findings, and typical neuroimaging results strongly suggestive of CNS infections. This study was approved by the Ethics Committee of PUMCH (S-K 807). Since this retrospective study was based on reviewing the medical records obtained for clinical purpose, the requirement of informed consent was waived and general confidential principles were obeyed.

\section{Study design \\ Demographics, clinical characteristics, and outcomes in SLE patients with CNS infection}

The medical records of these patients were systematically reviewed and evaluated by the MDT, and all clinical data was collected. Follow-up clinical evaluations were conducted in all available patients, to update the case outcomes. Demographic features, medical history, clinical manifestations, laboratory findings, previous treatments history including the use of GCs and DMARDs, and systemic lupus erythematosus disease activity index 2000 
(SLEDAI-2K) at the onset of CNS infection or NPSLE were recorded and presented in Table 1. All these patients underwent CSF examinations, including white blood cell (WBC) count and classification, and the protein, glucose, and chloride levels measurement. The etiological tests included microorganism culture; smear with gram, acid-fast, and India ink staining; CALAS test; and Mycobacterium tuberculosis DNA detection by PCR. Antibodies against viruses and parasites, such as Cysticercus cellulosae, were detected in cases as needed. Neuroimaging, such as magnetic resonance imaging (MRI), was conducted for all cases without contraindications. Otherwise, computed tomography $(\mathrm{CT})$ scans were performed.

\section{A simplified scoring system for discriminating CNS infections from NPSLE in SLE patients}

A simplified scoring system comprising 8 items was established for guiding clinical practice. Seventy-five out of 95 cases $(79 \%)$ among the CNS infections group and the NPSLE group were integrated to determine the risk factors. Four factors were concluded from a univariate analysis and fixed by further multivariate logistic stepwise regression with the cutoff values decided by the receiver operating characteristic (ROC) curve. The MDT then voted on the other items, and four criteria for the diagnosis of CNS infection vs. NPSLE, based on extensive clinical experience, were selected and cutoff values determined.

\section{Scoring system verification}

The scoring system was verified in the remaining 20 cases (21\%). Sensitivity and specificity were calculated, and the scoring system was optimized. The scoring system was then applied to 22 SLE patients who were admitted to PUMCH after January 2018, with a recent onset of neuropsychiatric symptoms, yet uncertain diagnosis of CNS infection or NPSLE. The final clinical diagnosis was traced and then compared to the predicted diagnosis based on our scoring model. Positive and negative predictive values were calculated for accuracy evaluation of prejudgement.

\section{Statistical analysis}

The chi-square $\left(\chi^{2}\right.$ test), Student $t$, and Mann-Whitney tests were used to compare the categorical data, numerical data with a normal distribution, and numerical data without a normal distribution, respectively. Data with a normal distribution are displayed with the mean and standard deviation (SD). Non-parametrially distributed data are represented as the median (interquartile range [IQR]). A univariate analysis was performed to determine the variables associated with CNS infections compared with NPSLE and factors associated with mortality. A multivariate logistic stepwise regression was performed with variables with $P$ value less than 0.05 in univariate analysis, a stepwise forward method used likelihood ratio test (LR) was used for variables entering the model. The ROC curve was utilized to find the cutoff values of variables with statistical significance for optimal event discrimination. Survival analysis was performed to compare the prognosis of the two groups using the Kaplan-Meier method calculated by log-rank test. The Statistical Package for the Social Sciences 20.0 (SPSS, Inc., Chicago, IL, USA) was used for all data analysis. A $P$ value less than 0.05 was considered statistically significant.

\section{Results}

Demographic, clinical features and etiological detection of SLE patients complicated with CNS infections

Of the 8491 SLE in-patients in PUMCH, 95 patients (1.12\%) were identified as having CNS infections, which is similar to what has been previously reported in the literature of $0.54-2.25 \%$ [11-13]. The female to male ratio of this cohort was 5.8:1 (81 vs. 14). The mean age at the time of SLE diagnosis was $31.0 \pm 13.9$ years, while the mean age at the onset of CNS infections was $34.6 \pm 13.7$ years. The median lupus disease duration prior to the onset of neuropsychiatric symptom in the CNS infections group was significantly longer than that in the NPSLE group (median 21.0 vs. 1.0 months, IQR $3-50$ vs. $0-22$ months, $P<0.001$ ). The median time interval from the onset of neuropsychiatric symptom to the diagnosis of CNS infections was 9.0 days (IQR 2.0-36.0 days) (Table 1).

Regarding previous treatments, $36.8 \%$ of patients in the CNS infections group had been treated with pulse GCs, in contrast to $7.4 \%$ of patients in the NPSLE group $(P<0.001)$. The daily dose of prednisone or equivalent in the past 6 months in the CNS infections group was much higher than that in the NPSLE group $(43.5 \pm 44.2$ $\mathrm{mg}$ vs. $21.8 \pm 37.5 \mathrm{mg}, P<0.001)$. Sixty-seven $(70.5 \%)$ patients in the CNS infections group had received at least one DMARD (including cyclophosphamide (CTX), mycophenolate mofetil (MMF), methotrexate, cyclosporin, tacrolimus, azathioprine, hydroxychloroquine, and leflunomide) in the past 6 months, in contrast to $36(37.9 \%)$ patients in the NPSLE group $(P<0.001)$ (Table 1$)$.

For the CNS infections group, the most common clinical manifestations at the onset of infection were fever (96.8\%), headache (89.5\%), and acute confusional state (51.6\%), all of which occurred more frequently than those in the NPSLE group $(23.2 \%, 44.2 \%, 20.0 \%$, respectively, all $P<0.001$ ) (Table 1). Lumbar puncture revealed that the CNS infections group had more severe intracranial hypertension, with 80 patients $(84.8 \%)$ having increased intracranial pressure (ICP) $\left(>180 \mathrm{mmH}_{2} \mathrm{O}\right.$, normal range $80-180 \mathrm{mmH}_{2} \mathrm{O}$ ) and $47(51.1 \%)$ having extremely high ICP (> $300 \mathrm{mmH}_{2} \mathrm{O}$ ), compared to $50.5 \%$ and 9.5\%, respectively, in the NPSLE group (both $P<0.001$ ). The median WBC count in the CSF and the proportion of polymorphonuclear (PMN) leukocyte were both expected 
Table 1 Baseline demographics, clinical features and treatments in SLE with infections vs. NPSLE

\begin{tabular}{|c|c|c|c|}
\hline Items & CNS infections $(n=95)$ & NPSLE $(n=95)$ & $P$ value \\
\hline Sex, female, $n(\%)$ & $81(85.3)$ & $81(85.3)$ & 1.000 \\
\hline Age at SLE diagnosis, year, mean (SD) & $31.0(13.9)$ & $30.8(14.1)$ & 0.897 \\
\hline Age at onset ${ }^{\S}$, years, mean (SD) & $34.6(13.7)$ & $32.3(14.7)$ & 0.276 \\
\hline SLE disease duration ${ }^{\&}$, months, median (IQR) & $21.0(3.0-50.0)$ & $1.0(0-22.0)$ & $<0.001$ \\
\hline \multicolumn{4}{|l|}{ System involvement of SLE, $n(\%)$} \\
\hline Lupus nephritis & $71(74.7)$ & $69(72.6)$ & 0.742 \\
\hline NPSLE & $26(27.4)$ & $95(100)$ & $<0.001$ \\
\hline Hematological & $65(68.4)$ & $62(65.3)$ & 0.644 \\
\hline Mucocutaneous & $79(83.2)$ & $66(69.5)$ & $<0.05$ \\
\hline Musculoskeletal & $51(53.7)$ & $52(54.7)$ & 0.884 \\
\hline Cardiovascular & $12(12.6)$ & $22(23.2)$ & 0.058 \\
\hline Pulmonary & $6(6.3)$ & $18(18.9)$ & $<0.05$ \\
\hline \multicolumn{4}{|l|}{ Medical history*, $n(\%)$} \\
\hline Pulmonary tuberculosis & $5(5.3)$ & $5(5.3)$ & 1.000 \\
\hline Fungal infections & $2(2.1)$ & $1(1.1)$ & 1.000 \\
\hline Diabetes mellitus & $9(9.5)$ & $4(4.2)$ & 0.151 \\
\hline Herpes zoster infections & $9(9.5)$ & $1(1.1)$ & $<0.01$ \\
\hline \multicolumn{4}{|l|}{ Previous treatment* } \\
\hline Pulse GCs, $n(\%)$ & $35(36.8)$ & $7(7.4)$ & $<0.001$ \\
\hline Average daily prednisone dose (or equivalent) in recent 6 months, mg/day, mean (SD) & $43.5(44.2)$ & $21.8(37.5)$ & $<0.001$ \\
\hline DMARDs in recent 6 months, $n(\%)$ & $67(70.5)$ & $36(37.9)$ & $<0.001$ \\
\hline CTX/MMF in recent 1 year, $n(\%)$ & $49(51.6)$ & $17(17.9)$ & $<0.001$ \\
\hline \multicolumn{4}{|l|}{ Neuropsychiatric symptoms ${ }^{\S}, n(\%)$} \\
\hline Fever & $92(96.8)$ & $22(23.2)$ & $<0.001$ \\
\hline Headache & $85(89.5)$ & $42(44.2)$ & $<0.001$ \\
\hline Seizure & $24(25.3)$ & $35(36.8)$ & 0.085 \\
\hline Psychosis & $17(17.9)$ & $31(32.6)$ & $<0.05$ \\
\hline Cognitive dysfunction & $17(17.9)$ & $32(33.7)$ & $<0.05$ \\
\hline Acute confusional state & $49(51.6)$ & $19(20.0)$ & $<0.001$ \\
\hline Anxiety disorder & $2(2.1)$ & $10(10.5)$ & $<0.05$ \\
\hline \multicolumn{4}{|l|}{ CSF examination $\$$} \\
\hline Pressure $\geq 300 \mathrm{mmH}_{2} \mathrm{O}, n(\%)$ & $47(51.1)$ & $9(9.5)$ & $<0.001$ \\
\hline WBCs, $10^{6} / \mathrm{L}$, mean (SD) & $635(1470)$ & $3(12)$ & $<0.001$ \\
\hline PMN ratio, \%, mean (SD) & $45.6(36.0)$ & $0.5(2.4)$ & $<0.001$ \\
\hline Protein, g/L, mean (SD) & $2.13(3.78)$ & $0.64(0.65)$ & $<0.001$ \\
\hline Glucose, mmol/L, mean (SD) & $2.0(1.3)$ & $3.3(0.9)$ & $<0.001$ \\
\hline \multicolumn{4}{|l|}{ Laboratory blood test at onset ${ }^{\S}$} \\
\hline WBCs, $10^{6} / \mathrm{L}$, mean (SD) & $9084(5898)$ & $6497(3508)$ & $<0.001$ \\
\hline PMN ratio, \%, mean (SD) & $82.2(10.3)$ & $75.8(11.9)$ & $<0.001$ \\
\hline Lymphocytes, $10^{6} / \mathrm{L}$, mean (SD) & $923(771)$ & $1032(758)$ & 0.354 \\
\hline Hypocomplementemia, n (\%) & $41(44.6)$ & $72(77.4)$ & $<0.001$ \\
\hline $\operatorname{lgG}, \mathrm{g} / \mathrm{L}$, mean $(\mathrm{SD})$ & $13.6(7.6)$ & $12.2(7.4)$ & 0.236 \\
\hline ESR, mm/h, mean (SD) & $54.8(39.5)$ & $48.2(31.8)$ & 0.215 \\
\hline SLEDAI-2K score, mean (SD) & $7.5(7.3)$ & $18.4(5.6)$ & $<0.001$ \\
\hline
\end{tabular}


Table 1 Baseline demographics, clinical features and treatments in SLE with infections vs. NPSLE (Continued)

\begin{tabular}{|c|c|c|c|}
\hline Items & CNS infections $(n=95)$ & NPSLE $(n=95)$ & $P$ value \\
\hline SLICC/ACR Damage Index, mean (SD) & $1.03(1.04)$ & $1.01(0.88)$ & 0.151 \\
\hline Morality rate ${ }^{\#}, n(\%)$ & $26(27.4)$ & $13(13.7)$ & $<0.05$ \\
\hline
\end{tabular}

Pulse GCs are defined as equal to or greater than $500 \mathrm{mg} /$ day methylprednisolone infusion for consecutive 3 5 days; DMARDs, including cyclophosphamide, mycophenolate mofetil, methotrexate, cyclosporin, tacrolimus, azathioprine, hydroxychloroquine, leflunomide

CNS central nervous system, GCs glucocorticoids, NPSLE neuropsychiatric lupus erythematosus, DMARDs disease-modifying antirheumatic drugs, CTX cyclophosphamide, MMF mycophenolate mofetil, CSF cerebrospinal fluid, ESR erythrocyte sedimentation rate, WBCS white blood cells, PMN polymorphonuclear leukocyte, IgG immunoglobulin G, SLEDAI-2K systemic lupus erythematosus disease activity index 2000. SLICC/ACR Systemic Lupus International Collaborating Clinics/American College of Rheumatology

${ }^{\S}$ Evaluated within 2 weeks of CNS infection or NPSLE onset

\&Disease duration from SLE diagnosis to CNS infections or NPSLE onset

*Evaluated history before the diagnosis of CNS infection or NPSLE

"Evaluated within 1 year of diagnosis of CNS infection or NPSLE

Significant $p$ values are shown in bold typeface

higher in the CNS infections group than those in the NPSLE group $\left(635 \pm 1470 / \mathrm{mm}^{3}\right.$ vs. $3 \pm 12 / \mathrm{mm}^{3}$ and $45.6 \pm 36.0 \%$ vs. $0.5 \pm 2.4 \%$, respectively, $P<0.05)$. Also, the CSF protein levels were elevated $(2.13 \pm 3.78$ vs. $0.64 \pm 0.65 \mathrm{~g} / \mathrm{L}$, $P<0.001)$ remarkably, and the glucose level decreased $(2.0 \pm 1.3$ vs. $3.3 \pm 0.9 \mathrm{mmol} / \mathrm{L}, P<0.001)$ significantly in the CNS infections group than in the NPSLE group (Table 1).

Etiological tests identified 63 well-defined pathogens in 59 patients (62.1\%), with 4 patients having been detected 2 pathogens at the same time. Common Bacteria were identified in 27 patients (45.8\%), followed by Cryptococcus in 18 patients (30.5\%) and mycobacteria in 11 patients (18.6\%). Among the bacterial infections, the top 3 species were Listeria monocytogenes in 10 patients (16.9\%), Nocardia asteroides in 4 patients (6.8\%), and staphylococci in 3 patients (5.1\%). Two patients (3.4\%) were identified as having viral meningoencephalitis: one was confirmed to have Epstein-Barr virus (EBV) meningoencephalitis with the evidence of high copies of EBV DNA in the CSF; the other was found to have high titers of anti-herpes simplex virus-IgM in the CSF. One patient $(1.7 \%)$ was diagnosed with cerebral cysticercosis, based on the positive anti-Cysticercus cellulosae antibody both in CSF and serum. Detailed pathogenic information for these patients was shown in Fig. 1.

Patients in the CNS infections group were followed up for $47 \pm 57$ months, but 18 patients (19\%) were lost to follow-up. Similarly, 19 patients $(20 \%)$ in the NPSLE group were lost to follow-up. Thirty patients $(31.6 \%)$ in the CNS infections group died during the follow-up, and 26 patients $(86.7 \%)$ died within the first year due to respiratory failure or cerebral hernia. Fourteen patients $(14.7 \%)$ in the NPSLE group died. Both the total and 1year mortality rates of the CNS infections group were

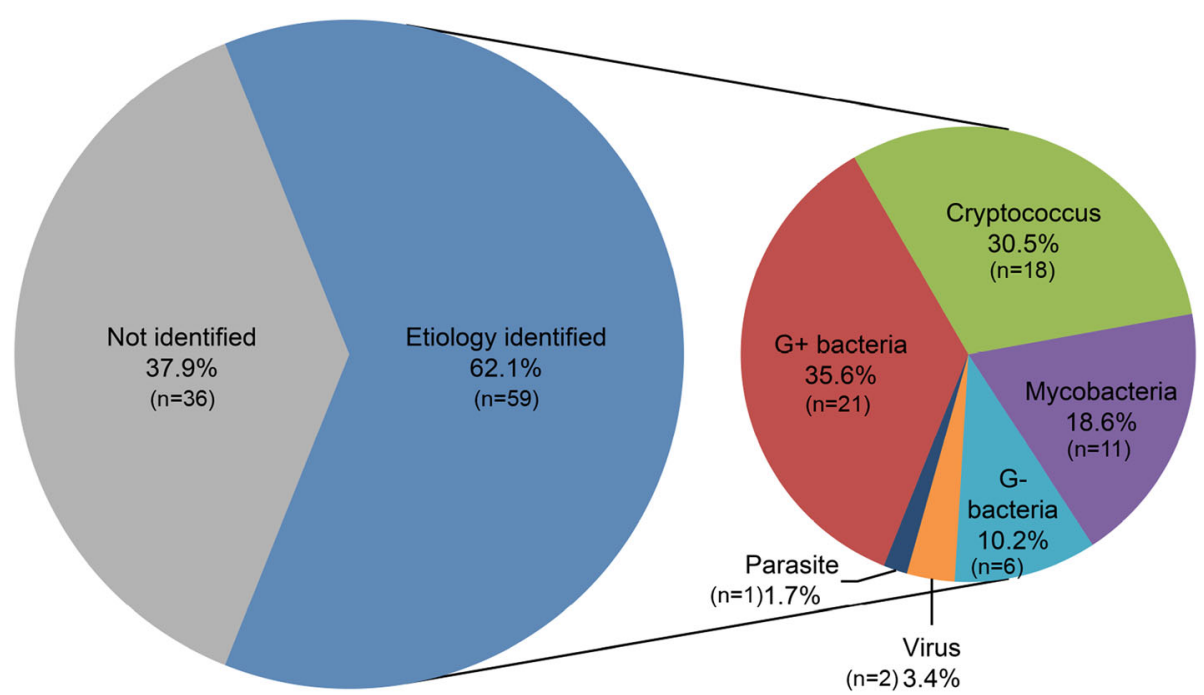

Fig. 1 The etiology of 59 SLE patients with CNS infections. The G+ bacteria included Listeria monocytogenes, Nocardia asteroides, Staphylococci epidermidis, Staphylococci hominis, Steptococcus pneumonia, Enterococcus faecium, Corynebacterium diphtheria and species undefined; G- bacteria included Pseudomonas aeruginosa, Acinetobacter Iwoffii, Acinetobacter Baumannii and species undefined; Virus included Epstein-Barr virus and Herpes simplex virus; Parasite referred Cysticercus botryoides. Species undefined, positive result of smear with gram staining but negative of microorganism culture; G+ bacteria, Gram-staining-positive bacteria; G- bacteria, Gram-staining-negative bacteria 

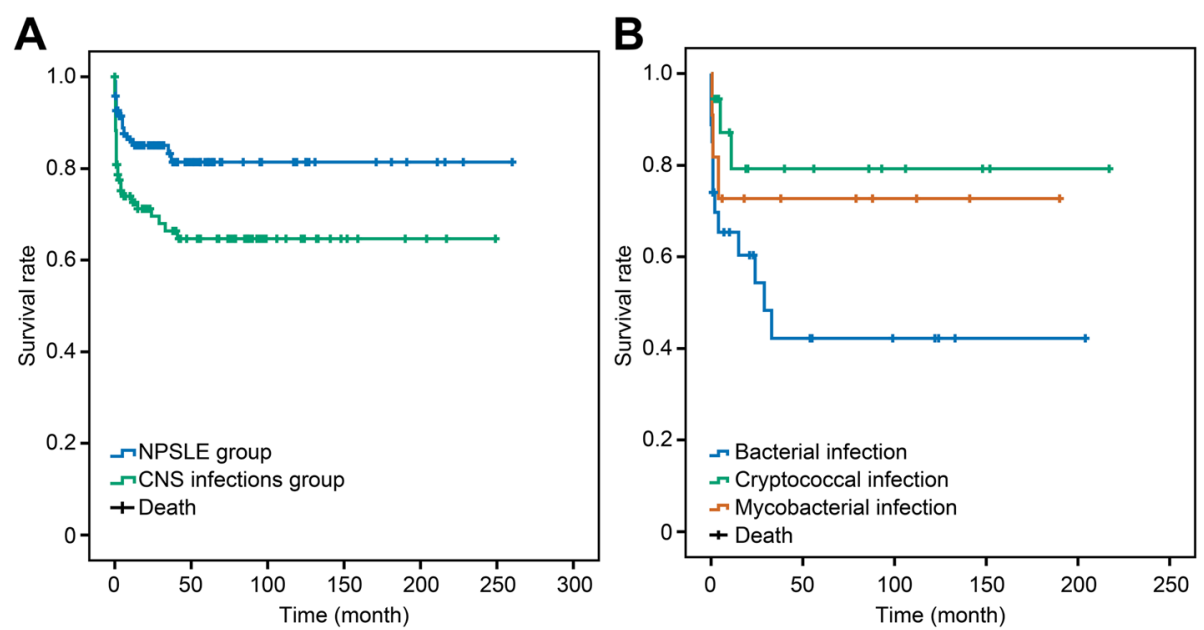

Fig. 2 Survival rates of SLE with CNS infections vs. NPSLE and etiology of CNS infections. a Survival rates of SLE patients with CNS infections and NPSLE. The survival rate of the CNS infections group was significantly lower than that of the NPSLE group $(P<0.01)$. $\mathbf{b}$ Survival rates of CNS infections in SLE patients with three different types of etiology. The survival rate of the bacterial subgroup was significantly lower than that of the other two subgroups, but the difference among the three groups was not statistically significant $(P=0.078)$. SLE, systemic lupus erythematosus; CNS, central nervous system; NPSLE, neuropsychiatric lupus

significantly higher than those of the NPSLE group (total mortality $31.6 \%$ vs. $14.7 \%$, 1 -year mortality $27.4 \%$ vs. $12.6 \%$, both $P<0.05$ ) (Fig. 2a).

The prevalence rate of CNS infections with SLE in our in-patient center with 5-year interval frame was $1.4 \% \pm$ $1.4 \%$, while the rate of NPSLE was $17.6 \% \pm 4.6 \%$ (Additional file 2). And the prevalence of CNS infections did not drastically change across this time frame, although there was an increase in the prevalence of NPSLE, from 13.1 to $21.4 \%$ during this time interval. The change of etiology for CNS infection in this cohort is shown in Additional file 3, where we can see that Gram-positive bacteria and Cryptococcus were the predominant pathogens throughout the whole time period.

\section{Diverse characteristics and prognosis in different CNS infections}

We categorized CNS infection patients into three subgroups according to their etiological findings as follows: bacterial $(n=27)$, cryptococcal $(n=18)$, and mycobacterial $(n=11)$ infections. Patients with bacterial or mycobacterial infections tended to manifest more consciousness disturbance $(59.3 \%, 54.5 \%$ vs. $16.7 \%, P<0.05)$ and meningeal irritation signs $(81.5 \%, 72.7 \%$ vs. $38.9 \%, P<0.05)$, compared to cryptococcal infections (Additional file 4). Nearly half of the total deaths $(13 / 30,43.3 \%)$ occurred in the bacterial infection subgroup. The first year mortality rate in the bacterial subgroup was higher than the other two subgroups (37.0\% vs. $16.7 \%$ and $27.3 \%)$. In the survival analysis, the bacterial subgroup showed the worst outcome among the three subgroups (Fig. 2b).

\section{Characteristics and prognosis of key points for the differential diagnosis of CNS infections from NPSLE} In the multivariate logistic regression analysis, compared with the NPSLE group, the CNS infections group had a longer disease duration (21.0 [3.0-50.0] vs.1.0 [0-22.0] months, $\mathrm{OR}=5.2,95 \% \mathrm{CI} 1.1-24.5, P<0.05)$, more frequent pyrexia $(96.8 \%$ vs. $23.2 \%, \mathrm{OR}=34.3$, 95\%CI $5.2-$ 226.7, $P<0.001)$, and PMN leukocytosis in the CSF (45.6\% vs. $0.5 \%, \mathrm{OR}=1.09$, 95\%CI $1.00-1.19, P<0.05)$ but significantly decreased CSF glucose ( $2.0 \pm 1.3$ vs. $3.3 \pm 0.9 \mathrm{mmol} / \mathrm{L}, \mathrm{OR}=13.7,95 \% \mathrm{CI} 2.1-85.8, P<0.01)$. Together, these results may be used as clues to distinguish CNS infections from NPSLE. Conversely, the existence of hypocomplementemia seemed to be a strong suggestive index of NPSLE over CNS infections (44.6\% vs. $77.4 \%$, $\mathrm{OR}=0.08,95 \%$ CI $0.02-0.41, P<0.01$ ) (Table 2 ).

Table 2 A multivariate logistic regression analysis of key points for discriminating CNS infections from NPSLE

\begin{tabular}{|c|c|c|c|}
\hline Variables & $P$ value & OR & $95 \% \mathrm{Cl}$ \\
\hline SLE disease duration ${ }^{\&} \geq 12$ months & $<0.05$ & 5.2 & $1.1-24.5$ \\
\hline Pulse GCs* & 0.070 & 7.7 & $0.8-70.7$ \\
\hline Fever $^{\S}$ & $<0.001$ & 34.3 & $5.2-226.7$ \\
\hline $\mathrm{CSF}$ glucose $\leq 2.2 \mathrm{mmol} / \mathrm{L}^{\S}$ & $<0.01$ & 13.7 & $2.1-85.8$ \\
\hline CSF PMN leukocytosis ${ }^{\S}$ & $<0.05$ & 1.10 & $1.00-1.19$ \\
\hline Hypocomplementemia ${ }^{\S}$ & $<0.01$ & 0.08 & $0.02-0.41$ \\
\hline
\end{tabular}

CNS central nervous system, NPSLE neuropsychiatric lupus erythematosus, GCs glucocorticoids, CSF cerebrospinal fluid, PMN polymorphonuclear leukocyte ${ }^{\&}$ Disease duration from SLE diagnosis to CNS infections or NPSLE onset *Evaluated medical history before the diagnosis of CNS infection or NPSLE ${ }^{5}$ Evaluated within 2 weeks of CNS infection or NPSLE onset

Significant $p$ values are shown in bold typeface 
Establishment of a simplified scoring system for discriminating CNS infections from NPSLE in SLE patients Through a univariate analysis and multivariate logistic stepwise regression, 4 items consisting of longer disease duration, fever, CSF, PMN ratio, significantly decreased CSF glucose, and absence of hypocomplementemia were established as the vital risk factors for discriminating CNS infections from NPSLE in SLE patients. CSF examinations, measuring intracranial pressure, WBC count, and protein levels, were also included for their crucial clinical significance evaluated by the MDT. As such, the above 8 items were integrated to establish a simplified scoring system, SSS-8, to assist the rapid recognition of CNS infection in SLE patients. Then, 75 out of 95 cases in the CNS infections group and NPSLE group were randomly selected and combined into one group, the establishment group, and the cutoff value for each item was decided via ROC. The remaining 20 cases were assigned as the verification group (Table 3 ).

The cutoff value of WBC in CSF, PMN ratio, CSF protein, and CSF glucose level in CSF were $20 / \mu \mathrm{L}, 0.5 \%, 0.905 \mathrm{~g} / \mathrm{L}$, and $2.2 \mathrm{mmol} / \mathrm{L}$, respectively (Fig. 3a) with the AUC being 0.88 (95\%CI 0.82-0.94), 0.88 (95\%CI 0.81-0.94), 0.88(95\%CI $0.82-0.93)$, and 0.80 (95\%CI $0.73-0.88)$, respectively. Notably, nearly $93 \%$ of patients in the cohort had normal blood glucose levels. An ICP over $300 \mathrm{mmH}_{2} \mathrm{O}$ and disease duration longer than 1 year were also considered significant (Tables 1 and 2). Thus, in SSS-8, the presence of each item was assigned to 1 point, and the highest score in SSS- 8 was 8 points (Table 3 ). A score equal to or above 4 points was indicative of a CNS infection with a sensitivity of $85 \%$ and specificity of $84.2 \%$ (Fig. 3).

\section{Verification of SSS-8 in SLE patients}

Verification of the SSS-8 was first done in the leftover retrospectively collected cases, referred to as the verification group $(n=20)$. With the cutoff value of 4 , a

Table 3 Simplified scoring system for distinguishing CNS infections from NPSLE

\begin{tabular}{|c|c|}
\hline Item & Score \\
\hline Disease duration ${ }^{\&} \geq 12$ months & 1 \\
\hline Fever $^{\S}$ & 1 \\
\hline Intracranial pressure $\geq 300 \mathrm{mmH}_{2} \mathrm{O}^{\S}$ & 1 \\
\hline WBCs in $C S F \geq 20 / \mu \mathrm{L}^{\S}$ & 1 \\
\hline PMN ratio in CSF $\geq 0.5 \%^{\S}$ & 1 \\
\hline Protein level in CSF $\geq 0.905 \mathrm{~g} / \mathrm{L}^{\S}$ & 1 \\
\hline Glucose level in CSF $\leq 2.2 \mathrm{mmol} / \mathrm{L}^{\S}$ & 1 \\
\hline Absence of hypocomplementemia & 1 \\
\hline Total & 8 \\
\hline
\end{tabular}

CNS central nervous system, NPSLE neuropsychiatric lupus erythematosus, CSF cerebrospinal fluid, $P M N$ polymorphonuclear leukocyte

${ }^{\&}$ Disease duration from SLE diagnosis to CNS infections or NPSLE onset

${ }^{\S}$ Evaluated within 2 weeks of CNS infection or NPSLE onset sensitivity of $85 \%$ and a specificity of $85.0 \%$ with the AUC being 0.93 (95\%CI 0.86-1.00) can be obtained for discriminating CNS infections from NPSLE (Fig. 3b).

We then verified SSS-8 in a group of SLE patients $(n=22)$ with a recent onset of neuropsychiatric symptoms with suspected CNS infection or NPSLE, who were admitted to PUMCH after January 2018. Patients in this cohort were scored using the SSS-8, and possible diagnosis was predicted according to SSS-8. Their final clinical diagnosis and etiology findings were traced and compared with the SSS- 8 prejudgment. In brief, seven patients were confirmed to be definitive CNS infection with etiology evidence obtained later; detailed information is exhibited in Table 4. AUC for SSS-8 in this cohort was 0.93 (95\%CI $0.80-1.00)$. The sensitivity and the specificity are $85.7 \%$ and $93.3 \%$, respectively. Positive predictive value and negative predictive value are $85.7 \%$ and $93.3 \%$, respectively (Fig. 3c).

\section{Discussion}

CNS infections are rare but a life-threatening complication of SLE. The incidence of CNS infections ranges from 0.54 to $2.26 \%$ according to the limited available reports [11-13]. In this study, based on a large cohort of patients from the tertiary referral center in China, we identified the incidence of CNS infections among SLE patients was $1.12 \%$. However, it should be noted that the investigation was conducted among in-patients in a single center without including out-patient data, due to the constraint of data integrity, and this may cause slight bias and limit the representation of the true incidence among the whole body of lupus patients in China. One strength of this study is that it is the first of its kind to use a sizable population cohort of more than 8000 SLE patients with comprehensive medical documents and reliable etiological evidence, all of which were carefully reviewed by a MDT. With the large sample size and direct comparison to symptoms of patients with NPSLE, this study highlights the general characteristics and outcomes of CNS infections among SLE patients and provides pivotal risk factors for differentiating CNS infections from NPSLE.

The pathophysiological factors that contribute to CNS infections in SLE patients are complicated and multifactorial. Vigorous treatment with immunosuppressants for controlling lupus disease may be one of the major causes of the susceptibility $[8,9,14,15]$. Our study reveals that $36.8 \%$ of SLE patients with CNS infections had been previously treated with pulse GCs, and roughly $50 \%$ of patients were treated with a powerful immunosuppressant like CTX and or MMF, within a year prior to CNS infections (in patients with NPSLE these are $7.4 \%$ and $17 \%$, respectively). The average daily dose of prednisone given to patients in the last 6 months of the study was 43.5 

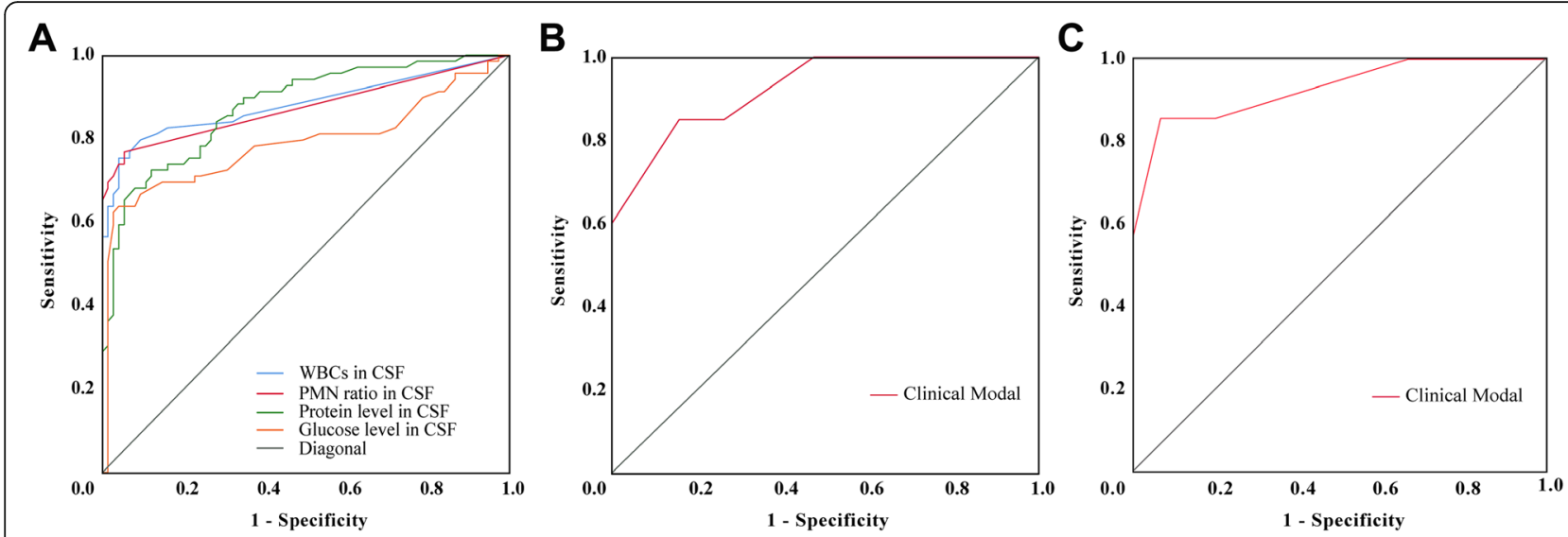

Fig. 3 ROC curve for WBC, PMN, glucose, protein in CSF, and SSS-8. a ROC curve for WBC, PMN, glucose, and protein in CSF with area under the curve (AUC) being 0.88 (95\% Cl 0.82-0.94), 0.88 (95\%Cl 0.81-0.94), $0.88(95 \% \mathrm{Cl} 0.82-0.93)$, and 0.80 (95\%Cl 0.73-0.88), respectively. The cutoff values for these four indexes are $20 / \mathrm{LL}, 0.5 \%, 0.905 \mathrm{~g} / \mathrm{L}$, and $2.2 \mathrm{mmol} / \mathrm{L}$, respectively. $\mathbf{b}$ ROC curve for simplified scoring system with 8 items (SSS-8). AUC is $0.93(95 \% \mathrm{Cl} 0.86-1.00)$. The cutoff value for distinguishing CNS infections and NPSLE is 4 with a sensitivity of $85.0 \%$ and a specificity of 85.0\%. c ROC curve for verifying SSS-8 in newly onset suspected CNS infection and NPSLE cases. The AUC is 0.93 (95\%Cl 0.80-1.00). The sensitivity and specificity are $85.7 \%$ and $93.3 \%$, respectively. ROC, receiver operating characteristic; WBC, white blood cell; CSF, cerebrospinal fluid; AUC, area under the curve; NPSLE, neuropsychiatric lupus erythematosus; PMN, polymorphonuclear leukocyte

$\mathrm{mg} /$ day in the CNS infections group, double the dosage given to patients in the NPSLE group, which was 21.8 $\mathrm{mg} /$ day. $70.5 \%$ patients in the CNS infections group had received at least one DMARD in the past 6 months, compared to $37.9 \%$ in the NPSLE group. Furthermore, defects in immune defense and surveillance in SLE may contribute to the susceptibility of infections and malignancy [16-18]. Our study finds that a small proportion of patients $(5,5.3 \%)$ had CNS infections at the onset of SLE, before they were given immunosuppressive treatment. On the other hand, infections may trigger a lupus flare by stimulating and activating the immune system. Evidence from the literature suggests that bacterial and viral infections might be involved in the induction, exacerbation, and/or flare of SLE $[16,18,19]$ by contributing to an aberrant immune response, predisposing to a tolerance breakdown toward native proteins [19]. Viruses such as EBV, cytomegalovirus, and Parvovirus B19 are frequently reported as environmental triggers in SLE autoimmunity [20-22], which may partly explain the collateral active lupus disease with bacterial/virus infection in our study.

In our cohort, Cryptococcus was the most common pathogen identified in SLE patients with CNS infections, followed by mycobacteria and Listeria monocytogenes, which is consistent with the previous reports $[11,13]$. Comparisons among these three categories of pathogens revealed that patients infected with Cryptococcus relatively had a better outcome (Fig. 2b), probably due to a chronic inclination in the disease course. Eleven patients had CNS infections with Mycobacterium confirmed by positive acid-fast bacilli (six patients), high-copy TB DNA (two patients), and culture (three patients). Mycobacterium tuberculosis is a type of intracellular bacteria. Impaired cell-mediated immunity, especially defects in the macrophages, renders susceptibility in SLE patients [23]. L. monocytogenes was the major pathogen identified in the bacterial infection subgroup. Patients with Gramnegative bacteria infection had the worst outcome, with a mortality rate of $83.3 \%$. Fifty percent of them died within 10 days after diagnosis, and the remaining $33.3 \%$ of patients died within 1 month.

Identifying biomarkers to assist the prompt diagnosis of infections and distinguish infections from a SLE flare is essential for preventing poor outcomes $[9,17,19,24,25]$. The anti-dsDNA antibody titer and the presence of hypocomplementemia have been proposed as biomarkers for lupus [26], as well as CD 64-Fc receptor expression [21, 27]. Other studies, looking at the prognostic factors of infections and SLE flares $[8,14]$ suggests that the administration of CTX and intravenous corticosteroids increase the risk of infections. However, when it comes to CNS infections, there may be other factors that are leading to the onset of infections. Our study reveals that besides a higher dose of daily corticosteroid treatment, and previous vigorous DMARD usage, patients who have a longer disease duration ( $\geq 1$ year), and patients with a previous history of herpes zoster infection, have a higher risk of CNS infections. This underscores the need for more intense monitoring of the primary disease during treatment and meticulous balance of the intensity of lupus treatment to minimize the risk of severe infection. Patients with neuropsychiatric symptoms, concurrent fever, persistent and violent headache, and consciousness disturbances, other than seizure disorders and cognitive dysfunction, should always be scrutinized for the possibility of CNS 


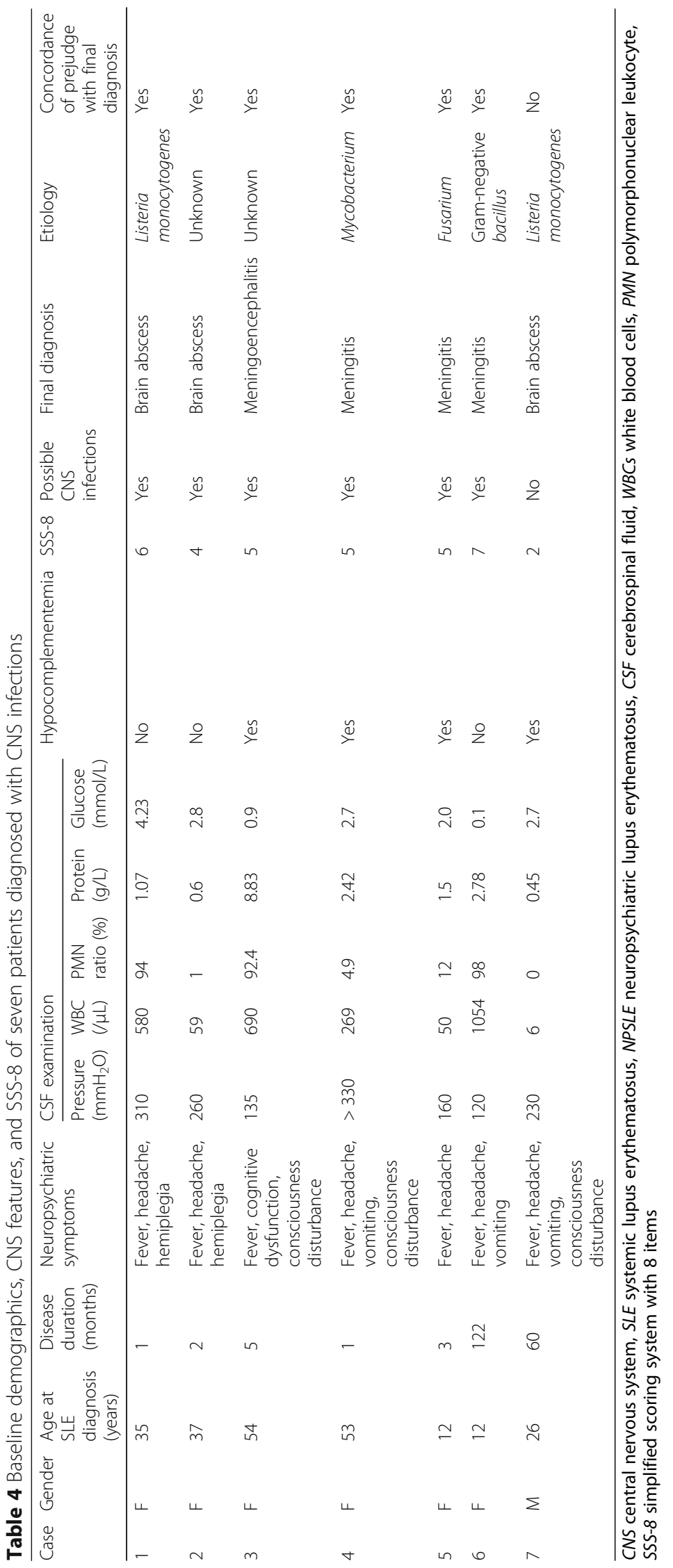


infections. Our data shows that CSF examination is extremely important. CSF test with extremely high intracranial pressure, leukocytosis predominant with PMN, and increased protein but decreased glucose levels is indicative of CNS infections instead of NPSLE.

We also aimed to construct a simplified and practical evaluation system to help prompt differentiation between these 2 severe and confusing complications, for doctors treating patients with SLE. By integrating 79\% SLE patients with CNS infections $(n=75)$ and NPSLE $(\mathrm{n}=75)$ via multivariate regression and ROC analysis, as well as suggestions made by a multiple disciplinary team, we generated an 8-item simplified scoring system, called SSS-8. The 8 items were a long disease duration $(\geq 1$ year), fever $\left(>37.3^{\circ} \mathrm{C}\right)$, absence of hypocomplementemia and super high intracranial pressure $\left(\geq 300 \mathrm{mmH}_{2} \mathrm{O}\right)$, CSF leukocytosis (WBC $\geq 20 / \mu \mathrm{L}$ ), PMN predominance in CSF $(\geq 0.5 \%)$, increased protein level $(\geq 0.905 \mathrm{~g} / \mathrm{L})$, and/ or decreased glucose level $(\leq 2.2 \mathrm{mmol} / \mathrm{L})$ in CSF. The presence of each item was assigned 1 point, and patients who scored 4 points or above were considered as more inclined to CNS infections. This scoring system was first verified in the remaining 20 cases of the retrospective SLE cohort and obtained an AUC of 0.93 (95\%CI 0.861.00 ) with a sensitivity and specificity for modal of $85.0 \%$ and $85.0 \%$, respectively. We then further verified the SSS-8 in a small group of SLE patients with a recent onset of neuropsychiatric symptoms and undetermined diagnosis of CNS infection or NPSLE. Their final clinical diagnosis was recorded and compared to their predicted diagnosis using the SSS- 8 scale. Our SSS- 8 scale evaluation predicted $6 / 7$ patients had CNS infections, and these cases were confirmed with etiologic evidence and/ or clinical decision. Further, the SSS-8 predicted 14/15 patients had non-CNS infections and turned out to have the final clinical diagnosis of NPSLE. However, team with limited number of experts, this scoring system does have some shortcomings: it is generated from a limited sample, and each case was discussed among a multidisciplinary team. Also, CSF lab tests are not sensitive for brain abscess which is not uncommon in CNS infections in SLE. Further verification in a large prospective cohort and optimization with better predictive markers or cutoff values are necessary before the widespread use of this rubric.

Primary NPSLE events, which account for $1 / 3$ of cases with neuropsychiatric manifestation in major SLE cohorts, are a consequence of either inflammatory mediators and autoantibodies, or thrombosis and microvasculopathy. The remaining $2 / 3$ of cases with neuropsychiatric manifestations are due to the secondary causes like treatment, infections, and metabolic abnormalities [28, 29]. To establish the diagnosis of NPSLE, causes other than SLE that might be responsible for the neuropsychiatric symptoms need to be excluded. In this study, we relied on ACR 1999 criteria and specialists' opinions for NPSLE classification and enrollment. And all NPSLE patients had received contemporary routine screening tests to rule out infections, for these patients might need to be treated promptly with high-dose or even pulse GC treatment. Case files were reviewed by two rheumatologists; also, a specialist of infectious disease was asked to join in for further exclusion of infection possibilities. We agree that although ACR1999 criteria were a milestone in the classification and nomenclature of NPSLE, limitations exist and confounders still remain [28]. In the future, the presence of NPSLE-associated autoantibodies, or cytokines in serum/ CSF, combined with MRI findings may add to the diagnosis criteria and contribute to a more accurate classification of NPSLE from other confounding illnesses.

\section{Conclusions}

Discrimination between central nervous system infections and SLE flares is extremely challenging in medical clinics. The proposed scoring system, SSS-8, which examines the disease duration, presence of fever, and absence of hypocomplementemia, together with CSF analysis showing extremely high intracranial pressure, high WBC levels predominant with PMN leukocyte, and high protein levels with a decrease in glucose levels, may help clinicians to promptly, and more adequately, distinguish CNS infections from NPSLE.

\section{Additional files}

Additional file 1: Flowchart of screening of SLE patients with CNS infections and NPSLE. (PDF $91 \mathrm{~kb}$ )

Additional file 2: The prevalence rate of CNS infections with SLE vs. NPSLE. (PDF $278 \mathrm{~kb}$ )

Additional file 3: The change of etiology of 59 SLE patients with CNS infections with a 5-year interval frame. (PDF 350 kb)

Additional file 4: CNS infections in SLE patients with different etiology findings. (PDF $66 \mathrm{~kb}$ )

\section{Abbreviations}

ACR: American College of Rheumatology; AUC: Area under the curve; CALAS: Cryptococcal Antigen Latex Agglutination System; CNS: Central nervous system; CSF: Cerebrospinal fluid; CT: Computed tomography; CTX: Cyclophosphamide; DMARDs: Disease-modifying antirheumatic drugs; EBV: Epstein-Barr virus; GCs: Glucocorticoids; MDT: Multiple disciplinary team; MMF: Mycophenolate mofetil; NPSLE: Neuropsychiatric lupus erythematosus; PCR: Polymerase chain reaction; PMN: Polymorphonuclear leukocyte; PNS: Peripheral nervous system; PUMCH: Peking Union Medical College Hospital; ROC: Receiver operating characteristic; SLE: Systemic lupus erythematosus; SLEDAI-2K: Systemic lupus erythematosus disease activity index 2000; SSS-8: Simplified scoring system with 8 items; WBC: White blood cell

\section{Acknowledgements}

We thank all the authors and patients contributing to this study.

\section{Authors' contributions}

JMD takes responsibility for the study design and conduct, data acquisition, integrity, analysis, and interpretation, writing and coordinating of the drafts 
of the report, and reviewing the report. ZLD contributed to the protocol development, study design and concept, supervision of the data collection, data interpretation, and critical writing and revision. ZX was involved in the protocol development, study design and concept, coordination and supervision of the data collection, critical revision and reviewing of the report, and quality checking. SXC, GX, NJW, and HXM contributed to the study concept, data interpretation, accuracy of data analysis, and revising of the report critically. All the authors approved the final version.

\section{Funding}

This work was supported by grants from the National Natural Science Foundation of China $(81788104,81630044,81601432,81771763$, and 91542000); Chinese Academy of Medical Science Innovation Fund for Medical Sciences (CIFMS2017-12 M-1-008, 2016-12 M-1-003, 2017-I2M-3-011, and 2016-12 M-1-008); National Key Research and Development Program: "Precise Medical Research" (2016YFC0903900); Natural Science Foundation of Beijing, China (7182129); and 2016 PUMC Hospital Fund for Junior Faculty (pumch-2016-2.1).

\section{Availability of data and materials}

The dataset used and/or analyzed during the current study is available from the corresponding authors on reasonable request.

\section{Ethics approval and consent to participate}

The study protocol was approved by the Institutional Ethics Committee of PUMC Hospital (S-K 807).

\section{Consent for publication}

Not applicable.

\section{Competing interests}

The authors declare that they have no competing interests.

\section{Author details}

'Department of Rheumatology and Clinical Immunology, Peking Union Medical College Hospital, Clinical Immunology Center, Chinese Academy of Medical Sciences and Peking Union Medical College, The Ministry of Education Key Laboratory, Beijing, China. ${ }^{2}$ Department of Infectious Disease, Peking Union Medical College Hospital, Chinese Academy of Medical Sciences and Peking Union Medical College, Beijing, China. ${ }^{3}$ Department of Radiology, Peking Union Medical College Hospital, Chinese Academy of Medical Sciences and Peking Union Medical College, Beijing, China. ${ }^{4}$ Department of Neurology, Peking Union Medical College Hospital, Chinese Academy of Medical Sciences and Peking Union Medical College, Beijing, China. ${ }^{5}$ Clinical Immunology Center, Peking Union Medical College Hospital, Chinese Academy of Medical Sciences and Peking Union Medical College Beijing, China.

\section{Received: 27 January 2019 Accepted: 2 August 2019}

Published online: 19 August 2019

\section{References}

1. Wallace DJ, Podell T, Weiner J, Klinenberg JR, Forouzesh S, Dubois EL. Systemic lupus erythematosus--survival patterns. Experience with 609 patients. Jama. 1981;245(9):934-8.

2. Ward MM, Pyun E, Studenski S. Long-term survival in systemic lupus erythematosus. Patient characteristics associated with poorer outcomes. Arthritis Rheum. 1995;38(2):274-83.

3. Moss KE, loannou Y, Sultan SM, Haq I, Isenberg DA. Outcome of a cohort of 300 patients with systemic lupus erythematosus attending a dedicated clinic for over two decades. Ann Rheum Dis. 2002;61 (5):409-13.

4. Thomas G, Mancini J, Jourde-Chiche N, et al. Mortality associated with systemic lupus erythematosus in France assessed by multiple-cause-ofdeath analysis. Arthritis Rheumatol. 2014;66(9):2503-11.

5. Juarez M, Misischia R, Alarcon GS. Infections in systemic connective tissue diseases: systemic lupus erythematosus, scleroderma, and polymyositis/ dermatomyositis. Rheum Dis Clin N Am. 2003;29(1):163-84.

6. Cervera R, Khamashta MA, Font J, et al. Morbidity and mortality in systemic lupus erythematosus during a 5 -year period. A multicenter prospective study of 1,000 patients. European Working Party on Systemic Lupus Erythematosus. Medicine. 1999;78(3):167-75.
7. Jacobsen S, Petersen J, Ullman S, et al. A multicentre study of 513 Danish patients with systemic lupus erythematosus. II. Disease mortality and clinical factors of prognostic value. Clin Rheumatol. 1998;17(6):478-84.

8. Noel V, Lortholary O, Casassus P, et al. Risk factors and prognostic influence of infection in a single cohort of 87 adults with systemic lupus erythematosus. Ann Rheum Dis. 2001;60(12):1141-4.

9. Rua-Figueroa I, Lopez-Longo J, Galindo-Izquierdo M, et al. Incidence, associated factors and clinical impact of severe infections in a large, multicentric cohort of patients with systemic lupus erythematosus. Semin Arthritis Rheum. 2017:47(1):38-45.

10. Liang Mh, Corzillius M, Bae SC, et al. The American College of Rheumatology nomenclature and case definitions for neuropsychiatric lupus syndromes. Arthritis Rheum. 1999:42:599-608

11. Hung JJ, Ou LS, Lee WI, Huang JL. Central nervous system infections in patients with systemic lupus erythematosus. J Rheumatol. 2005:32(1):40-3.

12. Yang $C D$, Wang $X D$, Ye $S$, et al. Clinical features, prognostic and risk factors of central nervous system infections in patients with systemic lupus erythematosus. Clin Rheumatol. 2007;26(6):895-901.

13. Vargas PJ, King G, Navarra SV. Central nervous system infections in Filipino patients with systemic lupus erythematosus. Int J Rheum Dis. 2009;12(3): 234-8.

14. Pryor BD, Bologna SG, Kahl LE. Risk factors for serious infection during treatment with cyclophosphamide and high-dose corticosteroids for systemic lupus erythematosus. Arthritis Rheum. 1996:39(9):1475-82.

15. Feldman $\mathrm{CH}$, Marty FM, Winkelmayer WC, et al. Comparative rates of serious infections among patients with systemic lupus erythematosus receiving immunosuppressive medications. Arthritis Rheum. 2017;69(2):387-97.

16. Zandman-Goddard G, Shoenfeld Y. Infections and SLE. Autoimmunity. 2005; 38(7):473-85.

17. Sciascia S, Ceberio L, Garcia-Fernandez C, Roccatello D, Karim Y, Cuadrado MJ. Systemic lupus erythematosus and infections: clinical importance of conventional and upcoming biomarkers. Autoimmun Rev. 2012;12(2):157-63.

18. Rigante D, Mazzoni MB, Esposito S. The cryptic interplay between systemic lupus erythematosus and infections. Autoimmun Rev. 2014;13(2):96-102.

19. Doria A, Canova M, Tonon M, et al. Infections as triggers and complications of systemic lupus erythematosus. Autoimmun Rev. 2008;8(1):24-8.

20. Poole BD, Templeton AK, Guthridge JM, Brown EJ, Harley JB, James JA. Aberrant Epstein-Barr viral infection in systemic lupus erythematosus. Autoimmun Rev. 2009;8(4):337-42.

21. Hsu TC, Tsay GJ. Human parvovirus B19 infection in patients with systemic lupus erythematosus. Rheumatology (Oxford). 2001;40(2):152-7.

22. Sekigawa I, Nawata M, Seta N, Yamada M, lida N, Hashimoto H. Cytomegalovirus infection in patients with systemic lupus erythematosus. Clin Exp Rheumatol. 2002;20(4):559-64.

23. Iliopoulos AG, Tsokos GC. Immunopathogenesis and spectrum of infections in systemic lupus erythematosus. Semin Arthritis Rheum. 1996;25(5):318-36.

24. Petri M. Infection in systemic lupus erythematosus. Rheum Dis Clin N Am. 1998:24(2):423-56.

25. Allen E, Bakke AC, Purtzer MZ, Deodhar A. Neutrophil CD64 expression: distinguishing acute inflammatory autoimmune disease from systemic infections. Ann Rheum Dis. 2002;61(6):522-5.

26. Beca S, Rodriguez-Pinto I, Alba MA, Cervera R, Espinosa G. Development and validation of a risk calculator to differentiate flares from infections in systemic lupus erythematosus patients with fever. Autoimmun Rev. 2015; 14(7):586-93.

27. Hussein OA, El-Toukhy MA, El-Rahman HS. Neutrophil CD64 expression in inflammatory autoimmune diseases: its value in distinguishing infection from disease flare. Immunol Investig. 2010;39(7):699-712.

28. Vivaldo JF, de Amorim JC, Julio PR, de Oliveira RJ, Appenzeller S. Definition of NPSLE: does the ACR nomenclature still hold? Front Med. 2018;5:138.

29. Hanly JG. Diagnosis and management of neuropsychiatric SLE. Nat Rev Rheumatol. 2014;10(6):338-47.

\section{Publisher's Note}

Springer Nature remains neutral with regard to jurisdictional claims in published maps and institutional affiliations. 